\title{
A novel set-based reachability method for optimal robust control of constrained linear systems
}

\author{
Riccardo Desimini and Maria Prandini
}

\begin{abstract}
This paper addresses finite horizon optimal control of linear dynamical systems affected by an additive bounded disturbance and subject to polyhedral state and input constraints. The goal is to design a static state feedback control law that minimizes a quadratic nominal cost while robustly satisfying the state/input constraints. We propose a novel set-based reachability approach and compare it against two alternative set-based approaches that were proposed for robust model predictive control (MPC) a few years back. All the three approaches offer a computational procedure to the design of a control policy, which is expressed as the sum of a state feedback term and an open loop term. While in the robust MPC methods the feedback term is a-priori fixed and only the open loop term is optimized, in the proposed method both of them are design parameters that are jointly optimally tuned. This is achieved by adopting a zonotopic parametrization of the control law that makes constraints and cost function respectively linear and quadratic in the parameters. As a result, the set-based reachability method provides a feasible solution for tighter constraints than the two alternative set-based methods.
\end{abstract}

\section{INTRODUCTION}

We consider a constrained control problem for a discrete time linear system affected by an additive bounded disturbance. Our goal is to design a static state feedback controller that minimizes the system deviation from a desired operating condition through the minimization of a quadratic cost function, while robustly enforcing given polyhedral state and input constraints.

We propose a novel solution that is inspired by the setbased reachability approach to control design recently introduced in [1]. The underlying idea behind set-based reachability analysis is to compute the sets of states that the system can reach during its evolution (reach sets) by propagating the set of initial states through the system dynamics, which can be affected by disturbances. The effectiveness of a setbased reachability approach in terms of both computability and conservativeness of the result depends on the adopted representation of the reach sets, jointly with the system dynamics through which reach sets evolve. In [1], the reach sets are represented through zonotopes, a special class of convex polytopes that are parameterized by a center and a finite set of generator vectors. They are closed under affine transformations and Minkowski sum, so that if the system dynamics is linear and the initial state and the system inputs take values in zonotopes, then, reach sets are zonotopes

R. Desimini and M. Prandini are with the Dipartimento di Elettronica Informazione e Bioingegneria, Politecnico di Milano, Piazza Leonardo da Vinci 32, 20133 Milano, Italy, email \{riccardo.desimini, maria.prandini\}@polimi.it as well and easily computable, even in the case of high dimensional systems, [2].

Inspired by [1], we adopt a parametrization of the state feedback control law in the form of a zonotope, which allows to compute exactly all (zonotopic) reach sets and to formulate the polyhedral state and input constraints as linear constraints in the design parameters. Differently from [1], center and generators of the zonotopic control policy are taken as design parameters and, in particular, generators providing the state feedback component of the control law are not constrained to be a linear combination of the generators of the (zonotopic) set representing the input constraints, but they are optimized.

From a computational perspective, our robust control design problem reduces to a finite convex optimization program with quadratic cost and linear constraints, which is easy to solve. We are thus able to overcome the key issue of lack of convexity that arises when optimizing over state feedback policies in robust constrained control (see e.g. [3]), which is often solved by optimizing only the open loop term and fixing a-priori a sensible value for the feedback term, [4] and [5]. Differently from [3], where convexity is recovered by using a control policy that depends affinely on all the past values of the state, we have a static state feedback control policy where the control input to be applied at a certain time instant depends on the state value at the same time instant.

Alternative parameterizations of the (static) state feedback law have been proposed in [6] and [7], where the vertices of a polytope are adopted in place of the center and generators of a zonotope. However, these parameterizations are less computationally convenient since, while the number of generators of a zonotope scales linearly with the space dimension, the number of vertices of a polytope grows exponentially with the space dimension, [1].

Similarly to [8], we compare the set-based computational approaches introduced in [4] and [5] with the proposed setbased reachability method. While the former two methods are feasible only with loose constraints due to the conservatism introduced to obtain computationally affordable procedures, our method does not introduce any conservatism when the disturbance takes value in a zonotope, and thus an effective solution can be obtained even when constraints are tight and other approaches turn out to be infeasible.

Admittedly, all the mentioned methods including the one proposed here compute a sub-optimal solution since they restrict the state feedback control law to a class of parameterized policies. Interestingly, this is not the case for the robust min-max approach proposed in [9], where the optimal piecewise affine state feedback policy is determined 
via dynamic programming. Computationally, the approach in [9] involves the solution of a set of multi-parametric linear programs and the resulting control law is defined on a polyhedral partition of the state space, which can be complicated, even for low dimensional systems.

\section{Basic notions and notations}

A (convex) polyhedron $\mathscr{P} \subset \mathbb{R}^{h}$ is defined as the intersection of $q$ half-spaces (H-representation [10]), and can be expressed through $P_{A} \in \mathbb{R}^{q \times h}$ and $p_{B} \in \mathbb{R}^{q}$ as $\mathscr{P}=$ $\left\{z \in \mathbb{R}^{h} \mid P_{A} z \leq p_{B}\right\}$ or $\mathscr{P}=\left(P_{A}, p_{B}\right)$ for ease of notation. A polytope is a (convex) bounded polyhedron. Zonotopes are centrally symmetric convex polytopes. More precisely, a convex polytope in $\mathbb{R}^{h}$ is called a zonotope if it can be written as $\mathscr{Z}=\left\{z \in \mathbb{R}^{h} \mid z=c+\sum_{i=1}^{r} \beta_{i} g_{i}, \beta_{i} \in[-1,1]\right\}$, where $c \in \mathbb{R}^{h}$ is the center and $g_{i} \in \mathbb{R}^{h}, i=1, \ldots, r$, are the generators. We shall then use $\langle c, G\rangle$ as a more concise notation of $\mathscr{Z}$, where $G \in \mathbb{R}^{h \times r}$ is the generator matrix, which contains the generators as its columns. A singleton $\{c\}$ with $c \in \mathbb{R}^{h}$ can be represented as $\langle c, G\rangle$ with $G \in \mathbb{R}^{h \times 0}$ that is the empty matrix. Given a generator matrix $G, G^{[k]}$ denotes its $k$-th column and $G^{[k, l]}$ denotes the submatrix composed by the generators of $G$ from column $k$ to column $l, l \geq k$.

\section{PROBLEM FORMULATION}

Consider a discrete time linear system with state $x \in \mathbb{R}^{n}$ evolving according to

$$
x_{t+1}=A x_{t}+B u_{t}+w_{t},
$$

starting from $x_{0}=\hat{x}_{0}$ at time $t=0$, subject to the control input $u \in \mathbb{R}^{m}$ and to the disturbance $w$ taking values in a zonotope $\mathscr{W}=\left\langle c_{w}, G_{w}\right\rangle$ with $c_{w}=0$ and $G_{w} \in \mathbb{R}^{n \times p_{w}}$.

Our goal is designing a static state feedback control law $\mu=\left(\mu_{0}, \ldots, \mu_{M-1}\right)$, where $\mu_{t}: \mathbb{R}^{n} \rightarrow \mathbb{R}^{m}, t=0, \ldots, M-1$, so that the closed-loop system

$$
\left\{\begin{array}{l}
x_{t+1}=A x_{t}+B u_{t}+w_{t} \\
u_{t}=\mu_{t}\left(x_{t}\right)
\end{array} \quad t=0, \ldots, M-1,\right.
$$

initialized at $x_{0}=\hat{x}_{0}$, satisfies the state and input constraints $x_{t} \in \mathscr{X}$ and $u_{t-1} \in \mathscr{U}, t=1, \ldots, M$, with $\mathscr{X} \subset \mathbb{R}^{n}$ that is a polyhedron, $\mathscr{U} \subset \mathbb{R}^{m}$ that is a polytope and both the two sets interiors contain the origin.

Among all feasible control laws, we select an optimal one according to the quadratic cost

$$
J_{\mu}=\sum_{t=0}^{M-1}\left(\bar{x}_{t+1}^{T} Q \bar{x}_{t+1}+\bar{u}_{t}^{T} R \bar{u}_{t}\right)
$$

associated with the nominal system

$$
\left\{\begin{array}{l}
\bar{x}_{t+1}=A \bar{x}_{t}+B \bar{u}_{t} \quad t=0, \ldots, M-1, \\
\bar{u}_{t}=\mu_{t}\left(\bar{x}_{t}\right)
\end{array} \quad t\right.
$$

representing the system behaviour when the disturbance $w$ is set equal to the center of the zonotope $\mathscr{W}$. Matrices $Q$ and $R$ are symmetric and positive semidefinite.

\footnotetext{
${ }^{1}$ If the disturbance $w$ takes values in a polytope, a zonotopic outerapproximation can be computed, [11], and its center can be shifted to the origin by a suitable change of coordinates.
}

The control design problem consists then in the following constrained optimization program:

$$
\begin{aligned}
& \min _{\substack{u_{t}=\mu_{t}\left(x_{t}\right), t=0, \ldots, M-1}} J_{\mu} \\
& \text { subject to: } \\
& \left\{\begin{array}{l}
x_{t} \in \mathscr{X}, t=1, \ldots, M \\
u_{t} \in \mathscr{U}, t=0, \ldots, M-1,
\end{array}\right.
\end{aligned}
$$

which is semi-infinite since the constraints are meant to hold robustly with respect to the disturbance $w_{t} \in \mathscr{W}, t=$ $0, \ldots, M-1$, entering the system dynamics (1).

\section{SET-BASED REACHABILITY CONTROL DESIGN}

In this section, we describe a novel set-based reachability method to solve (3), using a convenient parametrization of the state feedback control law. The proposed controller structure rests on the following result.

Proposition 1: Consider the control system

$$
\left\{\begin{array}{l}
x_{t+1}=A x_{t}+B u_{t}+w_{t} \\
u_{t}=c_{u, t}+G_{u, t} \alpha_{t}
\end{array}\right.
$$

initialized at time $t=0$ with $\hat{x}_{0}$, where the disturbance $w_{t}$ belongs to $\left\langle 0, G_{w}\right\rangle$ with $G_{w} \in \mathbb{R}^{n \times p_{w}}$ and the control input $u_{t}$ is defined through vector $c_{u, t} \in \mathbb{R}^{m}$, matrix $G_{u, t} \in \mathbb{R}^{m \times p_{t}}$ with $p_{t}=t p_{w}$, and vector $\alpha_{t} \in \mathbb{R}^{p_{t}}$ satisfying $\left\|\alpha_{t}\right\|_{\infty} \leq 1$. Then, it holds that $x_{1} \in\left\langle c_{x, 1}, G_{x, 1}\right\rangle$ and for all $t \geq 1$ there exists $\alpha_{t}$ that depends on $x_{t}$, such that $x_{t+1} \in\left\langle c_{x, t+1}, G_{x, t+1}\right\rangle$, where $c_{x, k}$ and $G_{x, k}, k \geq 1$, are given by:

$$
\begin{aligned}
& c_{x, k}=A^{k} \hat{x}_{0}+\sum_{j=0}^{k-1} A^{k-1-j} B c_{u, j} \\
& G_{x, k}=\left[A^{k-1} G_{w}+\sum_{j=0}^{k-2} A^{k-2-j} B G_{u, j+1}^{\left[1, p_{1}\right]}\right. \\
& \left.A^{k-2} G_{w}+\sum_{j=0}^{k-3} A^{k-3-j} B G_{u, j+2}^{\left[p_{1}+1, p_{2}\right]} \quad \ldots \quad G_{w}\right],
\end{aligned}
$$

with the understanding that summations ranging from $h_{1}$ to $h_{2}$ with $h_{1}>h_{2}$ give no contribution.

Moreover, a possible choice for $\alpha_{t}, t \geq 1$, is:

$$
\alpha_{t}^{*}=\underset{\alpha_{t} \in\left\{\beta_{t} \in \mathbb{R}^{p_{t}}: x_{t}=c_{x, t}+G_{x, t} \beta_{t},\left\|\beta_{t}\right\|_{\infty} \leq 1\right\}}{\arg \min }\left\|\alpha_{t}\right\|_{2}^{2}
$$

Proof: Since $w_{0} \in\left\langle 0, G_{w}\right\rangle$ and $u_{0}=c_{u, 0}$ (note that $G_{u, 0}$ is empty), the state of (4) at time $t=1$ is given by:

$$
x_{1}=A \hat{x}_{0}+B c_{u, 0}+G_{w} \alpha_{w, 0}, \quad\left\|\alpha_{w, 0}\right\|_{\infty} \leq 1
$$

so that $x_{1} \in\left\langle c_{x, 1}, G_{x, 1}\right\rangle$, where $c_{x, 1}=A \hat{x}_{0}+B c_{u, 0}$ and $G_{x, 1}=$ $G_{w}$ are given in (5) and (6) with $k=1$.

We prove the statement by induction on $t$, starting from $t=1$. Since $x_{1} \in\left\langle c_{x, 1}, G_{x, 1}\right\rangle$, we can choose $\alpha_{1}$ such that

$$
x_{1}=c_{x, 1}+G_{x, 1} \alpha_{1}, \quad\left\|\alpha_{1}\right\|_{\infty} \leq 1
$$

Note that we can set $\alpha_{1}$ to be an arbitrary value satisfying (8) and hence we can set it equal to the value in (7) with $t=1$. 
Now, by applying $u_{1}=c_{u, 1}+G_{u, 1} \alpha_{1}^{*}$, since $w_{1} \in\left\langle 0, G_{w}\right\rangle$, we obtain:

$$
\begin{aligned}
& x_{2}=A x_{1}+B u_{1}+w_{1}=A c_{x, 1}+B c_{u, 1}+ \\
& +\left[A G_{x, 1}+B G_{u, 1} \quad G_{w}\right]\left[\begin{array}{c}
\alpha_{1}^{*} \\
\alpha_{w, 1}
\end{array}\right], \quad\left\|\begin{array}{c}
\alpha_{1}^{*} \\
\alpha_{w, 1}
\end{array}\right\|_{\infty} \leq 1
\end{aligned}
$$

that is, $x_{2} \in\left\langle c_{x, 2}, G_{x, 2}\right\rangle$ where $c_{x, 2}=A c_{x, 1}+B c_{u, 1}$ and $G_{x, 2}=$ $\left[A G_{x, 1}+B G_{u, 1} \quad G_{w}\right]$ are given in (5) and (6) with $k=2$.

Now we prove that if the statement holds for $t-1$, then it holds also for $t$. By the induction hypothesis $x_{t} \in\left\langle c_{x, t}, G_{x, t}\right\rangle$, where the expressions of $c_{x, t}$ and $G_{x, t}$ are given in (5) and (6) by posing $k=t$. Now, by applying $u_{t}=c_{u, t}+G_{u, t} \alpha_{t}^{*}$, where $\alpha_{t}^{*}$ is given in (7), we obtain:

$$
\begin{aligned}
& x_{t+1}=A x_{t}+B u_{t}+w_{t}=A c_{x, t}+B c_{u, t}+ \\
& +\left[\begin{array}{ll}
A G_{x, t}+B G_{u, t} & G_{w}
\end{array}\right]\left[\begin{array}{c}
\alpha_{t}^{*} \\
\alpha_{w, t}
\end{array}\right], \quad\left\|\begin{array}{c}
\alpha_{t}^{*} \\
\alpha_{w, t}
\end{array}\right\|_{\infty} \leq 1
\end{aligned}
$$

that is, $x_{t+1} \in\left\langle c_{x, t+1}, G_{x, t+1}\right\rangle$ with $c_{x, t+1}=A c_{x, t}+B c_{u, t}$ and $G_{x, t+1}=\left[\begin{array}{ll}A G_{x, t}+B G_{u, t} & G_{w}\end{array}\right]$. By substituting $c_{x, t}$ and $G_{x, t}$ with their expressions obtained by applying the induction hypothesis, we finally obtain (5) and (6) for $k=t+1$.

Note that (7) works as a tie break rule and uniquely defines vector $\alpha_{t}$, with $\left\|\alpha_{t}\right\|_{\infty} \leq 1$, such that state $x_{t} \in\left\langle c_{x, t}, G_{x, t}\right\rangle$ is given by $x_{t}=c_{x, t}+G_{x, t} \alpha_{t}$.

By exploiting Proposition 1, we can parametrize the static state feedback control law $\mu=\left(\mu_{0}, \ldots, \mu_{M-1}\right)$ as follows:

$$
\left\{\begin{array}{l}
\mu_{0}(x)=c_{u, 0} \\
\mu_{t}(x)=c_{u, t}+G_{u, t} \alpha_{t}^{*}(x), t=1, \ldots, M-1
\end{array}\right.
$$

where

$$
\alpha_{t}^{*}(x)=\underset{\alpha \in\left\{\beta \in \mathbb{R}^{p_{t}}: x=c_{x, t}+G_{x, t} \beta,\|\beta\|_{\infty} \leq 1\right\}}{\arg \min }\|\alpha\|_{2}^{2}
$$

and $c_{x, t}$ and $G_{x, t}$ are given by (5) and (6) with $k=t$.

From the expressions in (9), it is clear that the proposed control law is composed by an open-loop term $c_{u, t}$ and a static state-feedback term $G_{u, t} \alpha_{t}^{*}(x)$, which will be jointly optimized compatibly with the constraints on state and input appearing in (3). Also, the control input $u_{t}$ belongs to the zonotope $\left\langle c_{u, t}, G_{u, t}\right\rangle$ which is optimized according to (3) and not structured as the constraint (zonotopic) set for the input as in [1].

In our set-up, the dynamics in (2) assumes the form

$$
c_{x, t+1}=A c_{x, t}+B c_{u, t}
$$

with $c_{x, 0}=\hat{x}_{0}$ and thus the cost in (3) can be rewritten as

$$
J_{\mu}=\sum_{t=0}^{M-1}\left(c_{x, t+1}^{T} Q c_{x, t+1}+c_{u, t}^{T} R c_{u, t}\right)
$$

Note that since $c_{x, t+1}, t=0, \ldots, M-1$, is linear as a function of the design variables $c_{u, k}, k=0, \ldots, t$ (see (5)), cost (10) is convex and quadratic in the optimization variables.

Our aim now is to show that the infinite number of constraints in (3) reduces to a finite number of linear inequalities in the control law parameters.
In order to enforce the state constraints at each time instant $t=1, \ldots, M$, we exploit the polytopic structure of the constraints set $\mathscr{X}$ and the fact that $x_{t} \in\left\langle c_{x, t}, G_{x, t}\right\rangle$ for all $t=1, \ldots, M$, as stated in Proposition 1 . Let $\left(X_{a}, x_{b}\right)$ be an H-representation of $\mathscr{X}$. Since $x_{t} \in\left\langle c_{x, t}, G_{x . t}\right\rangle$, we have that the constraint $x_{t} \in \mathscr{X}$ can be expressed as

$$
X_{a} c_{x, t}+\max _{\alpha \in[-1,1]^{t p_{w}}} X_{a} G_{x, t} \alpha \leq x_{b},
$$

where the $\max (\cdot)$ operator has to be applied elementwise, which is equivalent to the following constraint:

$$
X_{a} c_{x, t}+\left|X_{a} G_{x, t}\right| \mathbf{1}_{t p_{w}} \leq x_{b}
$$

where the operator $|\cdot|$ is meant to be applied elementwise and $\mathbf{1}_{d}$ denotes the $d$-dimensional column vector with unitary components. At time $t=1$, constraint (11) is simply

$$
X_{a} c_{x, 1}+\left|X_{a} G_{w}\right| \mathbf{1}_{p_{w}} \leq x_{b}
$$

and by using equation (5) it is readily obtained a linear inequality in the decision variable $c_{u, 0}$. At time $t=2, \ldots, M$, constraint (11) can be efficiently expressed as a set of linear inequalities in $c_{x, t}$ and $G_{x, t}$ by introducing suitable auxiliary variables, each one upper bounding an element of the absolute value matrix $\left|X_{a} G_{x, t}\right|$, and then plugging in these variables in (11). More precisely, we introduce the vectors $h_{x t j} \in \mathbb{R}^{q_{x}}, j=1, \ldots, p_{t-1}$ as:

$$
\begin{aligned}
& X_{a} c_{x, t}+\sum_{j=1}^{p_{t-1}} h_{x t j}+\left|X_{a} G_{w}\right| \mathbf{1}_{p_{w}} \leq x_{b} \\
& -h_{x t j} \leq X_{a} G_{x, t}^{[j]} \leq h_{x t j}, \quad j=1, \ldots, p_{t-1}
\end{aligned}
$$

Constraints (12) can be made explicit in terms of the decision variables $c_{u, 0}, c_{u, k}$ and $G_{u, k}, k=1, \ldots, t-1$ by using equations (5) and (6). A similar procedure applies also to the polytopic constraints on the control input, since the proposed control law has a zonotopic structure.

Summarizing, the resulting optimization problem is:

$$
\min _{\substack{c_{u, 0}, c_{u, t}, G_{u, t}, t=1, \ldots, M-1}} \sum_{t=0}^{M-1}\left(c_{x, t+1}^{T} Q c_{x, t+1}+c_{u, t}^{T} R c_{u, t}\right)
$$

$$
\text { subject to: }
$$

$$
\left\{\begin{array}{l}
X_{a} c_{x, t}+\left|X_{a} G_{x, t}\right| \mathbf{1}_{t p_{w}} \leq x_{b}, t=1, \ldots, M \\
U_{a} c_{u, 0} \leq u_{b} \\
U_{a} c_{u, t}+\left|U_{a} G_{u, t}\right| \mathbf{1}_{t p_{w}} \leq u_{b}, t=1, \ldots, M-1,
\end{array}\right.
$$

where we omitted the auxiliary variables for ease of reading.

\section{SET-BASED CONTROL DESIGN FOR ROBUST MPC}

\section{A. Approach based on invariant set}

We next briefly describe the approach based on the computation of a disturbance invariant set to bound the uncertain dynamics of (1) that was originally introduced in [4].

The control law has the following structure:

$$
u_{t}=K\left(x_{t}-\tilde{x}_{t}\right)+c_{t},
$$

where $K$ is an a-priori fixed stabilizing gain (e.g., the optimal gain of the infinite horizon unconstrained LQ control 
problem), while the open loop term $c_{t}$ is the actual decision variable. As for $\tilde{x}_{t}$ appearing in (14), it is governed by

$$
\tilde{x}_{t+1}=A \tilde{x}_{t}+B c_{t}
$$

and its initialization $\tilde{x}_{0}$ is a decision variable too. By plugging (14) into (1), the system dynamics becomes

$$
x_{t+1}=(A+B K) x_{t}-B K \tilde{x}_{t}+B c_{t}+w_{t} .
$$

If we now consider the difference $e$ between the actual dynamics and the one in (15), i.e., $e=x-\tilde{x}$, it satisfies the equation

$$
e_{t+1}=(A+B K) e_{t}+w_{t} .
$$

Now, denote by $\mathscr{Z}$ a disturbance invariant set for system (16), that is a set that satisfies the property that if $e_{t} \in \mathscr{Z}$, then $e_{t+1}$ must belong to $\mathscr{Z}$ for every realization of the disturbance $w_{t}$ with support $\mathscr{W}$. Based on the properties of invariant sets, it is immediately verified that if $\hat{x}_{0} \in \tilde{x}_{0} \oplus \mathscr{Z}$, then $x_{t+1} \in \tilde{x}_{t+1} \oplus \mathscr{Z}$ and $u_{t} \in c_{t}+K \mathscr{Z}$ (note that $K \mathscr{Z}=$ $\{K z, z \in \mathscr{Z}\}), t=0, \ldots, M-1$. In other words, $\mathscr{Z}$ can be used to bound the uncertain dynamics of (1).

The robust constraints in (3) can then be enforced by selecting $\tilde{x}_{0}$ and $c_{0}, \ldots, c_{M-1}$ so that $\hat{x}_{0} \in \tilde{x}_{0} \oplus \mathscr{Z}, \tilde{x}_{t} \oplus \mathscr{Z} \subseteq \mathscr{X}$, $t=1, \ldots, M$ and $c_{t} \oplus K \mathscr{Z} \subseteq \mathscr{U}, t=0, \ldots, M-1$. This leads to the following optimization problem:

$$
\min _{\tilde{x}_{0}, c_{t}, t=0, \ldots, M-1} \sum_{t=0}^{M-1}\left(\tilde{x}_{t+1}^{T} Q \tilde{x}_{t+1}+c_{t}^{T} R c_{t}\right)
$$

subject to:

$$
\left\{\begin{array}{l}
\hat{x}_{0} \in \tilde{x}_{0} \oplus \mathscr{Z} \\
\tilde{x}_{t} \oplus \mathscr{Z} \subseteq \mathscr{X}, t=1, \ldots, M \\
c_{t} \oplus K \mathscr{Z} \subseteq \mathscr{U}, t=0, \ldots, M-1 .
\end{array}\right.
$$

If the invariant set $\mathscr{Z}$ is a polytope, then problem (17) can be solved at relatively low computational cost, since the constraints can be enforced for the vertices of $\mathscr{Z}$ and $K \mathscr{Z}$ only. The invariant set $\mathscr{Z}$ should be as small as possible so as to achieve the widest feasibility for (17). The minimal invariant set, however, may not be a polytope, [4], and it is also difficult to compute. Usually an invariant polytopic outer approximation of the minimal invariant set is used, [12].

\section{B. Tube-based approach}

The tube-based approach developed in [5] for the case of multiplicative uncertainty is here formulated for the case of additive uncertainty by following the same approach of [8]. Specifically, the control law is selected as:

$$
u_{t}=K \bar{x}_{t}+L\left(x_{t}-\bar{x}_{t}\right)+c_{t}
$$

where the decision variable is the open loop term $c_{t}$. The state $x$ is split into the sum of a nominal term, represented by $\bar{x}$, and an error term, represented by $e=x-\bar{x}$. The dynamics of $\bar{x}$ and $e$ are described by the equations

$$
\begin{aligned}
& \bar{x}_{t+1}=(A+B K) \bar{x}_{t}+B c_{t}, \bar{x}_{0}=\hat{x}_{0} \\
& e_{t+1}=(A+B L) e_{t}+w_{t}, e_{0}=0 .
\end{aligned}
$$

Note that the first equation is obtained from (2) by setting $\mu_{t}\left(\bar{x}_{t}\right)=K \bar{x}_{t}+c_{t}$, which is (18) when $x_{t}=\bar{x}_{t}$.

The matrices $K$ and $L$ in (18) are some a-priori fixed gains used to stabilize the nominal and the error dynamics respectively. The idea is to find at each time step $t$ in the control horizon a polytope $\mathscr{P}_{t}$ that includes any possible value of $e_{t}$ when $w_{t-1}$ ranges over $\mathscr{W}$. The evolution of $x$ is then guaranteed to be contained in $\bar{x}_{t} \oplus \mathscr{P}_{t}$, while $u_{t}$ takes values in $K \bar{x}_{t}+c_{t} \oplus L \mathscr{P}_{t}$. The satisfaction of the robust constraints in (3) is guaranteed by requiring that these polytopes are subsets of $\mathscr{X}$ and $\mathscr{U}$, respectively. The final optimization problem is:

$$
\min _{c_{t}, t=0, \ldots, M-1} \sum_{t=0}^{M-1}\left(\bar{x}_{t+1}^{T} Q \bar{x}_{t+1}+\left(K \bar{x}_{t}+c_{t}\right)^{T} R\left(K \bar{x}_{t}+c_{t}\right)\right)
$$

subject to:

$$
\left\{\begin{array}{l}
\bar{x}_{t} \oplus \mathscr{P}_{t} \subseteq \mathscr{X}, t=1, \ldots, M \\
K \bar{x}_{t}+c_{t} \oplus L \mathscr{P}_{t} \subseteq \mathscr{U}, t=0, \ldots, M-1 .
\end{array}\right.
$$

Since $\mathscr{P}_{t}$ is a polytope, constraints in (19) can be enforced for the vertices of $\mathscr{P}_{t}$ and $L \mathscr{P}_{t}$ only, and problem (19) can be solved through standard convex optimization techniques.

As for the computation of $\mathscr{P}_{t}, t=0, \ldots, M$, they are selected in the form $\mathscr{P}_{t}=\left\{e_{t}: V e_{t} \leq \gamma_{t}\right\}$, where inequality is understood componentwise. The matrix $V$ has to be apriori fixed, which causes the facets of $\mathscr{P}_{t}$ to have always the same orientation for every $t=0, \ldots, M$. Vector $\gamma_{t}$ defines the distance between the facets at time $t$ so as to bound the possible values for $e_{t}$.

Since $\mathscr{W}$ is a polytope, one can determine $\mathscr{P}_{0}, \ldots, \mathscr{P}_{M}$ as follows: first, it is selected $\mathscr{P}_{0}=\{0\}$, which implies $\gamma_{0}=0$. Then, the matrix $H$ with all positive elements that satisfies $H V=V(A+B L)$ while minimizing the trace of $H H^{T}$ is computed and finally $\gamma_{1}, \ldots, \gamma_{M}$ are determined so as to satisfy $H \gamma_{t} \leq \gamma_{t+1}-V v_{w j}$ for all $t=0, \ldots, M-1$ and for all $j=1, \ldots, n_{v w}$, with $v_{w j}$ denoting the $j$-th vertex of $\mathscr{W}$. Further details are reported in [8].

\section{NUMERICAL EXAMPLES}

In this section, we make a comparative analysis of the approaches in Sections III and IV, in terms of their capability of providing an effective solution to the optimal constrained control problem described in Section II as the state and input constraints become tighter and tighter. To this purpose, we revisit the two numerical examples in [8]: a second order system, first, and, then, a fourth order spring-mass system.

\section{A. Second order system}

We consider a second order system described by:

$$
x_{t+1}=\left[\begin{array}{cc}
0.5 & -0.5 \\
0.5 & 0.5
\end{array}\right] x_{t}+\left[\begin{array}{l}
1 \\
0
\end{array}\right] u_{t}+w_{t}
$$

where $w$ is an additive disturbance with support $\mathscr{W}=$ $[-0.2,0.2]^{2}$. 
The finite horizon problem to be solved takes the form:

$$
\min _{\substack{u_{t}=\mu_{t}\left(x_{t}\right), t=0, \ldots, M-1}} \sum_{t=0}^{M-1}\left(\bar{x}_{t+1}^{T} Q \bar{x}_{t+1}+\bar{u}_{t}^{T} R \bar{u}_{t}\right)
$$

subject to:

$$
\left\{\begin{array}{ll}
\left\|u_{t}\right\|_{\infty} \leq u_{\text {lim }} & t=0, \ldots, M-1 \\
\left\|C x_{t}\right\|_{\infty} \leq y_{\text {lim }} & t=1, \ldots, M
\end{array},\right.
$$

where we set $M=9, Q=I_{2}, R=0.1, C=I_{2}$. As for the apriori fixed quantities entering the solution of (21) according to the approaches of Section IV:

- the feedback gains $K$ in (14) and $K, L$ in (18) are set equal to the optimal LQ gain $K_{L Q}$;

- the shaping matrix $V$ of the tube-based approach is

$$
V=\left[\begin{array}{cccccccc}
1 & 0 & -1 & 0 & 1 & 1 & -1 & -1 \\
0 & 1 & 0 & -1 & 1 & -1 & 1 & -1
\end{array}\right]^{T} .
$$

We set $x_{0}=\hat{x}_{0}=0$ and assess the possible conservativeness of the three approaches by evaluating the threshold values $y_{t h r}$ and $u_{t h r}$ for $y_{l i m}$ and $u_{l i m}$ in (21) leading to infeasibility of the optimization problems (13), (17) and (19). Results are shown in Table I.

TABLE I

ESTIMATE OF THE THRESHOLD VALUES $y_{t h r}$ AND $u_{t h r}$ FOR $y_{l i m}$ AND $u_{l i m}$ BEFORE INCURRING IN INFEASIBILITY.

\begin{tabular}{r|cc} 
& $y_{t h r}$ & $u_{t h r}$ \\
\hline Tube-based approach & 0.60 & 0.39 \\
\hline Approach based on invariant set & 0.53 & 0.36 \\
\hline Set-based reachability approach & 0.40 & 0.40
\end{tabular}

Note that, when the initial state is zero, the optimization problem (17) in the approach of Section IV-A is feasible if and only if the invariant set $\mathscr{Z}$ and its projection $K \mathscr{Z}$ on the input space through the feedback gain $K$ are respectively contained in the constraints sets $\mathscr{X}$ and $\mathscr{U}$. Hence, the thresholds $y_{t h r}$ and $u_{t h r}$ can be obtained based on $\mathscr{Z}$ and $K \mathscr{Z}$. Likewise, when the initial state is zero, problem (19) in the approach of Section IV-B is feasible if only if the tube sections $\mathscr{P}_{t}$ and $L \mathscr{P}_{t}$ are contained in the state and input constraints sets for every $t$. Since $\mathscr{P}_{t}$ and $L \mathscr{P}_{t}$ are increasing with $t, y_{t h r}$ and $u_{t h r}$ are obtained based on the tube sections $\mathscr{P}_{M}$ and $L \mathscr{P}_{M-1}$.

Since the reach sets shape is not a-priori defined, but is optimized when designing the control law, the values reported in Table I for the set-based reachability method are obtained by first monitoring the feasibility of problem (21) when progressively reducing $y_{\text {lim }}$ while taking a fixed high value of $u_{l i m}$, and then, once the threshold value for $y_{\text {lim }}$ has been detected, by progressively decrease $u_{\text {lim }}$ until problem (21) becomes infeasible. In this way, we first assess the capability of the controller to keep the reach sets inside the state constraints set when it can apply arbitrarily large control actions, and then, by keeping fixed the computed lower bound for $y_{\text {lim }}$, we assess the controller performance by progressively reducing its actuation capabilities.
Note that if $y_{\text {lim }}$ in (21) is set equal to the threshold values 0.60 and 0.53 reported in Table I for the tube-based approach and the approach based on invariant set, $u_{\text {lim }}$ can be decreased up to $u_{t h r}=0.11$ and $u_{t h r}=0.17$, respectively, before problem (21) becomes infeasible for the set-based reachability method. Both these threshold values for $u_{l i m}$ are much lower than those of the other two methods.

As it appears, the set-based reachability approach provides tighter constraints thresholds with respect to the other approaches. This is due to the fact that it is computing exactly the reach sets while also tuning the state feedback term, which results in a less conservative approach. In Figure 1 are shown the computed sets when feasibility is achieved only with the set-based reachability method: here, the shape of the reach sets is adapted by the controller in such a way to fit the tight state constraints, while the sets $\mathscr{Z}$ and $\mathscr{P}_{t}$, since have fixed structure, cannot be included into the state constraints box in the considered time horizon.

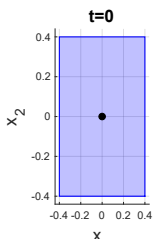

$x_{1}$
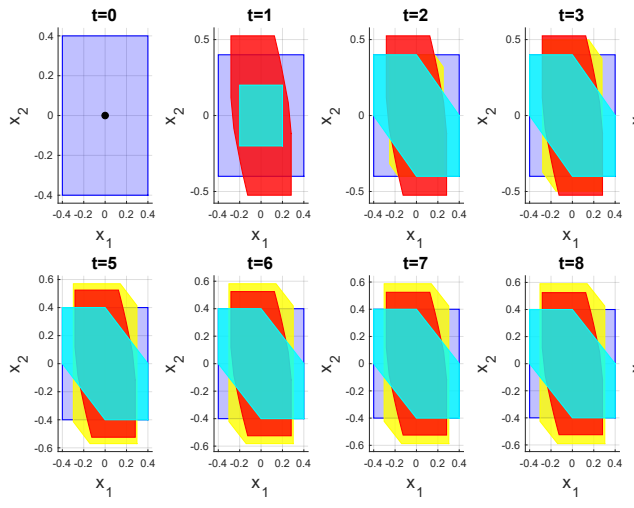

$x_{1}$
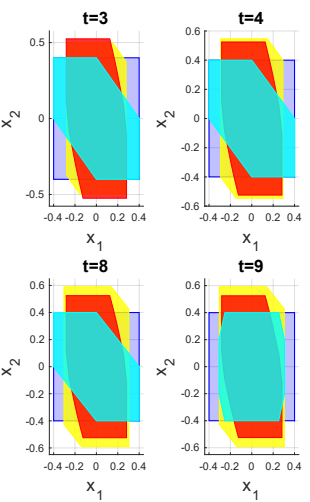

$x_{1}$

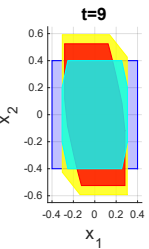

Fig. 1. Invariant set $\mathscr{Z}$ (red), sections of the tube (yellow) and reach sets obtained with the proposed method (cyan) when $y_{\text {lim }}=u_{\text {lim }}=0.4$.

Note that the feasibility of problems (17) and (19) depends on the choice of the feedback gains $K$ in (14) and $K, L$ in (18). It may be that choices other than $K_{L Q}$ lead to smaller thresholds than those reported in Table I. However, $K$ in (17) and $K, L$ in (19) have to be fixed in advance, otherwise computational difficulties arise, and it is usually difficult to guess what is the best choice.

\section{B. Spring-mass system}

We next present the simulation results obtained by applying the three approaches to the mechanical system composed of two masses and two springs shown in Figure 2.

The 4-dimensional state of the system is given by mass displacements with respect to an equilibrium point and their derivatives, whereas the control input is given by two forces acting on the masses. The system is discretized with standard techniques assuming constant input between consecutive time steps. All stiffnesses and masses have been chosen unitary. The disturbance added to the discrete time state has 


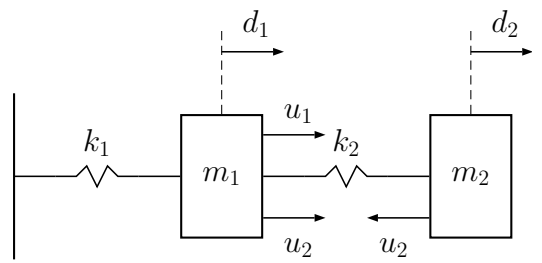

Fig. 2. Scheme of the mechanical system.

support $\mathscr{W}=[-1,1]^{4}$. The finite horizon control problem takes the form (21) with the following parameter settings:

$$
M=5, \quad Q=\left[\begin{array}{cc}
I_{2} & 0 \\
0 & 0
\end{array}\right], \quad R=I_{2}, \quad C=\left[\begin{array}{cccc}
1 & 0 & 0 & 0 \\
-1 & 1 & 0 & 0
\end{array}\right] .
$$

In particular, $C$ appearing in the state constraints is chosen so that $y_{\text {lim }}$ in (21) represents some bound posed on the deformation of both springs. The shape of the tube sections used in the approach of Section IV-B is a 4-dimensional hyper-rectangle: $V=\left[\begin{array}{ll}I_{4} & -I_{4}\end{array}\right]^{T}$.

As in the previous example, we first determine how much we can tighten the constraints before reaching infeasibility for the three approaches. Also in this case the set-based reachability approach provides tighter constraints than the other two approaches (see Table II).

TABLE II

ESTIMATE OF THE THRESHOLD VALUES $y_{t h r}$ AND $u_{t h r}$ FOR $y_{l i m}$ AND $u_{l i m}$ BEFORE INCURRING IN INFEASIBILITY.

\begin{tabular}{r|rr} 
& $y_{t h r}$ & $u_{t h r}$ \\
\hline Tube-based approach & 22.52 & 12.65 \\
\hline Approach based on invariant set & 5.94 & 4.57 \\
\hline Set-based reachability approach & 2.00 & 9.93
\end{tabular}

The tube-based approach turns out to be much more conservative than the one based on invariant set and the setbased reachability method.

Note that if $y_{\text {lim }}$ in (21) is set equal to the threshold value 5.94 reported in Table II for the approach based on invariant set, $u_{\text {lim }}$ can be decreased up to $u_{t h r}=0.85$ before problem (21) becomes infeasible for the set-based reachability method.

The performances obtained with tight constraints $\left(y_{\text {lim }}=2\right.$ and $u_{\text {lim }}=9.93$ ) are depicted in Figure 3, where the sets computed with the three approaches are represented in the output space.

\section{CONCLUSiOnS}

In this paper we addressed optimal quadratic constrained control for a linear system subject to a bounded additive disturbance. We proposed a novel computational approach and compared our method with two alternative set-based approaches that have been proposed in the literature on robust MPC some years back, showing by means of two examples taken from [8] that our method is better performing when state constraints are tight. This enhances the use of setbased reachability control within robust MPC, and opens

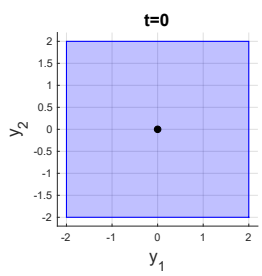

$\mathrm{t}=3$
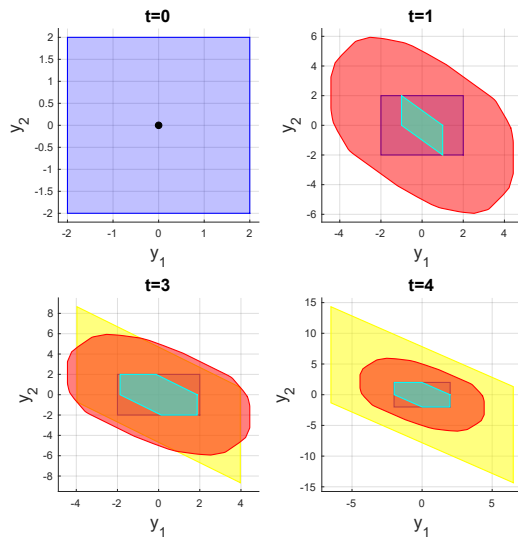

$\mathrm{t}=4$
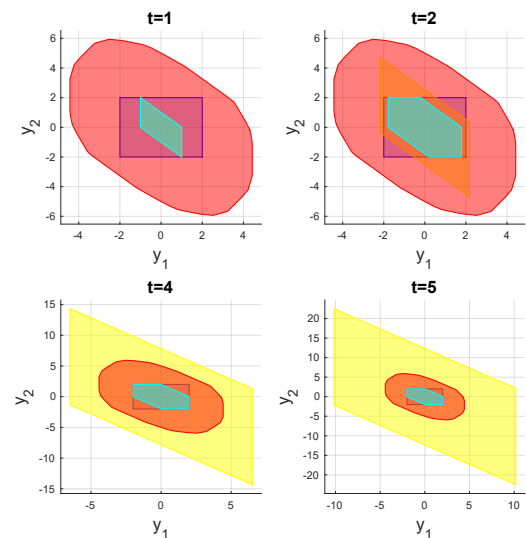

Fig. 3. Output space projections through matrix $C$ of the invariant set $\mathscr{Z}$ (red), of the sections of the tube (yellow) and of the reach sets obtained with the proposed method (cyan) when $y_{\text {lim }}=2$ and $u_{\text {lim }}=9.93$.

new interesting research directions related to the study of its recursive feasibility properties.

\section{REFERENCES}

[1] B. Schürmann and M. Althoff, "Guaranteeing constraints of disturbed nonlinear systems using set-based optimal control in generator space," IFAC-PapersOnLine, vol. 50, no. 1, pp. 11 515-11 522, 2017.

[2] A. Girard, "Reachability of uncertain linear systems using zonotopes," in Hybrid Systems: Computation and Control, M. Morari and L. Thiele, Eds. Berlin, Heidelberg: Springer Berlin Heidelberg, 2005, pp. 291-305.

[3] P. J. Goulart, E. C. Kerrigan, and J. M. Maciejowski, "Optimization over state feedback policies for robust control with constraints,' Automatica, vol. 42, no. 4, pp. 523-533, 2006.

[4] D. Q. Mayne, M. M. Seron, and S. V. Raković, "Robust model predictive control of constrained linear systems with bounded disturbances," Automatica, vol. 41, no. 2, pp. 219-224, 2005.

[5] M. Evans, M. Cannon, and B. Kouvaritakis, "Robust MPC for linear systems with bounded multiplicative uncertainty," in 2012 IEEE 51st IEEE Conference on Decision and Control (CDC), Dec. 2012, pp 248-253.

[6] B. Schürmann and M. Althoff, "Convex interpolation control with formal guarantees for disturbed and constrained nonlinear systems," in Proceedings of the 20th International Conference on Hybrid Systems. Computation and Control. New York, NY, USA: ACM, 2017, pp. 121-130.

[7] P. O. Gutman and M. Cwikel, "Admissible sets and feedback control for discrete-time linear dynamical systems with bounded controls and states," in The 23rd IEEE Conference on Decision and Control, Dec. 1984, pp. 1727-1731

[8] L. Deori, S. Garatti, and M. Prandini, "Computational approaches to robust model predictive control: a comparative analysis," IFAC Proceedings Volumes, vol. 47, no. 3, pp. 10 820-10 825, 2014.

[9] A. Bemporad, F. Borrelli, and M. Morari, "Min-max control of constrained uncertain discrete-time linear systems," IEEE Transactions on Automatic Control, vol. 48, no. 9, pp. 1600-1606, 2003.

[10] G. M. Ziegler, Lectures on polytopes, ser. Graduate Texts in Mathematics. New York, NY, USA: Springer-Verlag New York, 2012, vol. 152.

[11] M. Althoff, "Reachability analysis and its application to the safety assessment of autonomous cars," Ph.D. dissertation, Technische Universität München, München, Germany, July 2010.

[12] S. V. Raković, E. C. Kerrigan, K. I. Kouramas, and D. Q. Mayne, "Invariant approximations of the minimal robust positively invariant set," IEEE Transactions on Automatic Control, vol. 50, no. 3, pp. 406410, 2005. 